\title{
Description de la femelle de Culicoides congolensis Clastrier, 1960
}

\author{
Par M. KREMER et J. CALLOT
}

A notre demande, notre Collègue et ami le Professeur Larivière, que nous remercions bien vivement, nous a fait parvenir de Dakar des prélèvements de boues, en vue d'y rechercher des Cératopogonidés.

Parmi les prélèvements effectués, au nombre de six, un seul a donné lieu à des éclosions de Culicoides. Il s'agissait de boues provenant du bord d'un fossé de la ferme de l'Institut Pasteur à Mbao-Niaye (Sénégal). Le prélèvement avait été fait le 18 mai 1963. Les éclosions ont eu lieu à partir du 17 juin suivant.

Il s'agissait d'un Culicoides dont le mâle, remarquable par son hypopygium, a été décrit par Clastrier en 1960 au Congo et dont la femelle était demeurée inconnue.

\section{CULICOIDES CONGOLENSIS Clastrier, 1960}

Mâle : La seule différence avec la description de Clastrier porte sur la coloration du thorax que nous avons pu examiner à l'état frais : sa couleur générale est brunâtre ; quatre bandes percurrentes plus foncées antéro-postérieures latérales (deux de chaque côté du scutum, deux un peu plus en dehors) ; une bande sombre médiane part du bord antérieur du scutum mais s'arrête entre les taches réniformes sans atteindre le bord postérieur du scutum. Une bande sombre, perpendiculaire aux précédentes, traverse le scutum en son milieu et présente des élargissements aux points d'intersections.

Femelle : Aspect général identique à celui du mâle.

Tête: Yeux séparés par un espace très étroit allant en s'élargissant vers le front ; un poil inséré entre les yeux dans cet élargissement ; pas de pubescence oculaire.

Mandibules et maxilles denticulées.

Palpes de couleur générale blanchâtre clair. Deux premiers articles cylindriques, troisième article losangique, étroit, dont tout un bord est occupé par une fossette sensorielle remplie de poils et d'organes en massue. Quatrième article globuleux ; cinquième légèrement plus allongé. Longueur des articles (les deux premiers comptés ensemble) : $45,6 / 41,8 / 19 / 22,8 \mu$. 


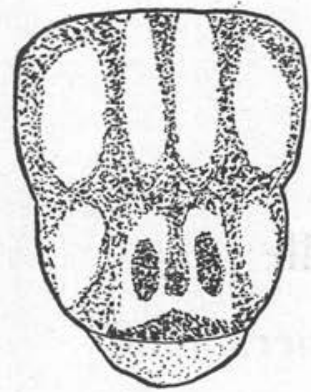

FIG. 1. - Culicoïdes congolensis $\&$ : thorax
Antennes légèrement plus sombres que les palpes; quatre premiers articles nettement plus sombres et chitinisés que les suivants; premier article du flagelle claviforme, les suivants globuleux, un peu renflés à la base, s'allongeant progressivement du $3^{\circ}$ au $10^{\circ}$; les cinq articles distaux en forme de pomme de pin.

Quand il n'y a pas de sensilles sur un article, on voit sur la face opposée à celle où ils devraient se trouver deux poils courts, épais et rectilignes, en plus des soies habituelles.

Indice antennaire : 1,31 .

DisPosition DES SENSILLES ET LONGUeUR DES ARTICLES DU FLAGELLE

\begin{tabular}{|c|c|c|c|c|c|c|c|c|c|c|c|c|c|}
\hline Articles..... & 3 & 4 & 5 & 6 & 7 & 8 & 9 & 10 & 11 & 12 & 13 & 14 & 15 \\
\hline $\begin{array}{l}\text { Sensilles (nom- } \\
\text { bre) } \ldots . . \ldots \ldots \ldots\end{array}$ & 3 & 0 & 1 & 0 & 1 & 0 & 1 & 1 & 1 & 1 & 1 & 1 & 1 \\
\hline Longueur en $\mu$ & 41,8 & 22,8 & 22,8 & 26,6 & 26,6 & 26,6 & 26,6 & 26,6 & 51,3 & 49,4 & 53,2 & 55,1 & 79,8 \\
\hline
\end{tabular}
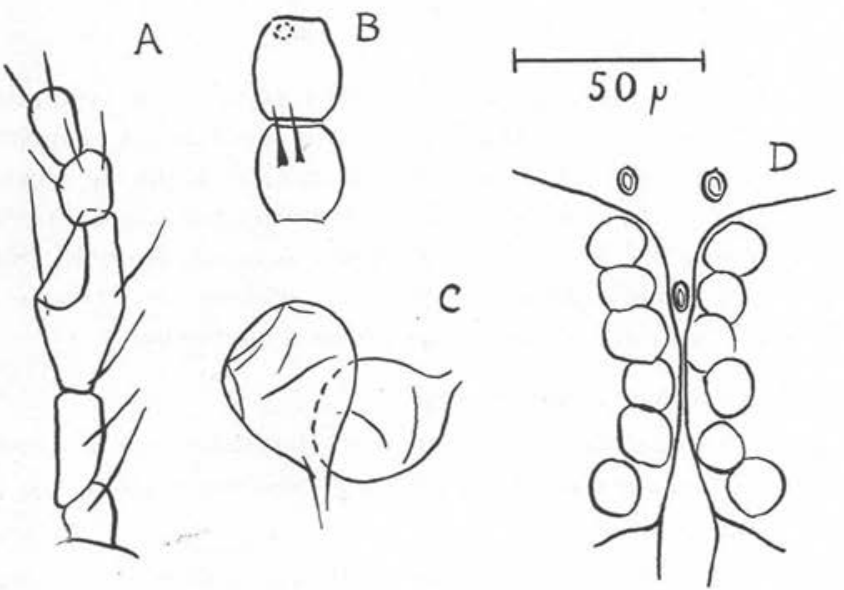

FIG. 2. $-C$. congolensis $\$$ : A, palpe; B, articles du flagelle antennaire avec ou sans sensilles; $C$, spermathèques; $D$, espace interoculaire

tailles décroissantes, dont les longueurs sont environ : 55, 42, 34, $30 \mu$.
Aile : elle est semblable à celle de Culicoides similis comme le signale Clastrier ; macrotriches un peu plus abondantes que chez le mâle. Longueur de l'aile (à partir de l'arculus) : $0,84 \mathrm{~mm}$; costa : $0,48 \mathrm{~mm}$; largeur : 0,41 $\mathrm{mm}$.

Thorax: comme celui du mâle.

Pattes : comme celles du mâle. Peigne tibial à quatre soies principales, de 
Abdomen: légèrement brunâtre; spermathèques peu chitinisées, globuleuses, se prolongeant par un col dont la limite avec les conduits spermatiques est mal définie. Diamètre des spermathèques 38 et $42 \mu$, sans le col. On peut voir un anneau chitinisé mais pas de spermathèque accessoire.

Allotype et deux mâles déposés dans la collection de l'Institut de Parasitologie de Strasbourg.

\section{Bibliographie}

Clastrier (J.), 1960. - Notes sur les Cératopogonidés. IX. Cératopogonidés de la République du Congo. Arch. Inst. Pasteur Algérie, 38, 79-105.

\section{Faculté de Médecine de Strasbourg, Institut de Parasitologie}

(Directeur : Professeur J. CaLLot)

Erratum à la note « Nouvelles espèces et nouvelles localisations de Culicoides... », par J. Callot, M. Krémer et Y. Déduit. Ces Annales, tome 37, 1962, p. 153-171.

Une erreur a fait inverser les légendes de certaines figures : la figure 3, page 159, concerne Culicoides jurensis; la figure 6, page 162, concerne Culicoides clastrieri. 